\title{
ENDODONTIC TREATMENT WITH MTA IN A TOOTH WITH OPEN APEX- A CASE REPORT
}

\author{
Dr. Vandana ${ }^{1}$, Dr. Ruchi Singla ${ }^{2}$, Dr. Jagat Bhushan ${ }^{3}$ \\ ${ }^{1}$ Lecturer, Department of Conservative Dentistry \& Endodontics Dr. HSJ Dental College, Panjab University, Chandigarh (India) \\ ${ }^{2}$ Sr. Lecturer, Department of Conservative Dentistry \& Endodontics Dr. HSJ Dental College, Panjab University, Chandigarh (India) \\ ${ }^{3}$ Prof. \& Head Department of Conservative Dentistry \& Endodontics Dr. HSJ Dental College, Panjab University, Chandigarh (India) \\ Corresponding Author: \\ ${ }^{3}$ Mobile: 919855442735 Email: drjagat@ gmail.com
}

\begin{abstract}
Received :

$7^{\text {th }}$ April, 2013

Accepted:

$10^{\text {th }}$ July, 2013

Available online:

$25^{\text {th }}$ August, 2013

This case report describes the treatment of a tooth with necrotic pulp and open apex (maxillary left central incisor). In this conventional multiple-visit apexification with calcium hydroxide was replaced with a simple two step apical barrier techinique using MTA. The treatment involved mild debridement of the dentinal walls with K files and repeated irrigation with sodium hypochlorite solution, followed by calcium hydroxide dressing for 1 week and lastly creating an apical plug with MTA. The objective of the treatment was to provide an apical stop for the root canal filling material.

Keywords: Tooth apex, Root canal obturation, Mineral trioxide aggregate
\end{abstract}

\section{INTRODUCTION}

Endodontic management of the non-vital permanent teeth with a wide open apex has long presented a challenge to dentistry. In these teeth thin, fragile dentinal walls made it difficult to achieve an apical seal. Many techniques have been advocated to manage these teeth. The most widely accepted technique is cleaning and filling the canal with a temporary paste to stimulate the formation of calcified tissue at the apex. The term apexification is used to describe this procedure. Calcium hydroxide apexification has been used successfully for years but has many disadvantages which include higher patient compliance, multiple appointments extending over a long period and time, unpredictability of apical closure and potential for coronal leakage or tooth fracture exists. ${ }^{1}$

An alternate approach is the placement of an apical barrier to prevent extrusion of the root filling. Materials considered for barriers include dentine chips, freeze dried cortical bone/ dentin, calcium phosphate and calcium hydroxide. The main advantage with this technique is the efficiency of creating a barrier and obturation in one appointment, but these materials do not provide a well-sealed apical environment. ${ }^{2}$
To overcome disadvantages of above materials, Torabinejad and Chivian in 1996 introduced the use of mineral trioxide aggregate (MTA) as an apical plug. ${ }^{3}$ MTA has been proposed as a potential material to create an apical plug at the end of the root-canal system, thus preventing the extrusion of filling materials. ${ }^{4}$ In this article we report the clinical application of MTA as an apical plug.

\section{CASE REPORT}

A 36 year old female patient came to the department with a discoloured maxillary left central incisor. Dental history revealed that the patient had a traumatic injury around $15 y$ rs back. During intraoral examination, it was found that 21 was discolored with an intact crown. The tooth responded normally to percussion, palpation and had normal findings during periodontal probing and mobility. Periapical radiograph showed an open apex with periapical radioluency (Figure 1).

After the application of the rubber dam, access cavity was prepared without anaesthesia. Radiographs were used to confirm the working length of the canal, which came out to be $18 \mathrm{~mm}$. At this stage, the number 80 file was found loose and was easily passing beyond the apical limit of the canal. The 
canal was lightly debrided and cleaned by using intracanal instruments and irrigants $\mathrm{NaOCl} 5 \%$ solution. To obtain canal disinfection prior to MTA placement, canal was dried with paper points, calcium hydroxide mixed with sterile water was placed with lentulospiral in the canal, and the access was closed with restorative material IRM (Caulk, Dentsply, Milford, DE). After 1week the patient was recalled and calcium hydroxide was removed by repeated rinsing with $\mathrm{NaOCl} 5 \%$ followed by rinsing with sterile water. Grey ProRoot MTA (Maillfer, Dentsply, Switzerland) was then mixed with distilled water according to manufacturer's instructions and delivered to the canal using an amalgam carrier and condensed to the apical end with pluggers. This procedure was repeated a number of times until the thickness of MTA reached almost 5mm. The plug's position in canal was checked by radiographs (Figure 2), to confirm the exact proximity and length of apical plug.

A wet cotton pellet with sterile water was placed in the canal and the access cavity was closed with temporary filling material (IRM). After two weeks, the filling material and the cotton pellet were removed and the set of the MTA was gently tested. The rest of the canal was then obturated with guttapercha by lateral condensation technique (Figure 3 ). The tooth was coronally sealed with composite resin.

Six months follow up demonstrated a radiographic decrease in the periapical radiolucency. The tooth was asymptomatic and clinically functional(Figure 4).

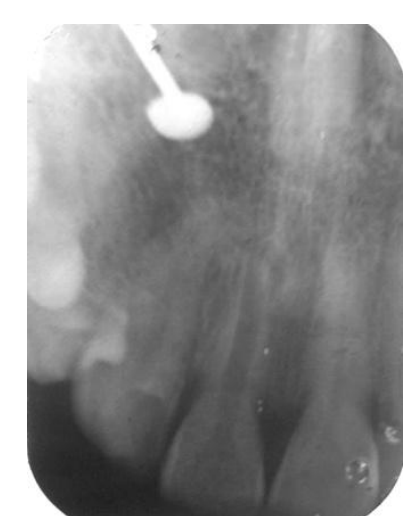

Figure 1: Preoperative radiograph of maxillary left central incisor with open apex

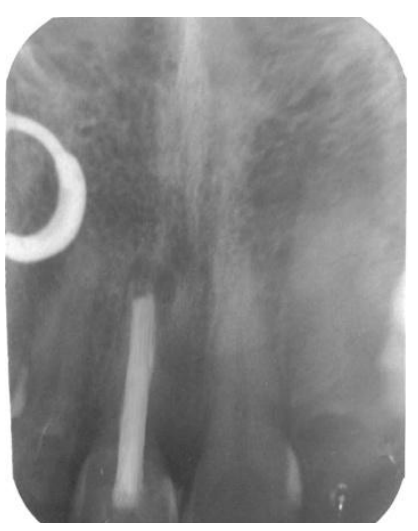

Figure 3: Postoperative radiograph

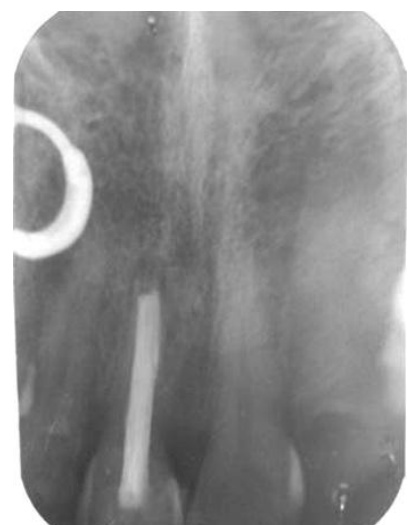

Figure 4: Follow up after 6 months

\section{DISCUSSION}

Mineral Trioxide Aggregate (MTA) is a cement type material that was introduced into the field of endodontics in 1993 by Loma Linda University. ${ }^{5}$ It has been used worldwide, with several clinical applications such as apical barriers in teeth with immature apices, repair of root perforations, direct pulp capping, pulpotomy and root canal filling material. In vitro studies have demonstrated its capacity to stimulate cytokine release and interleukin production from bone cells, indicating that this material actively promote hard tissue formation. ${ }^{6}$ Several authors have suggested that MTA may be ideal material that consistently allows overgrowth for the cementum like and bone like tissues and may facilitate regeneration of periodontal fibres. ${ }^{7}$

The principal components present in the mineral trioxide material are tricalcium silicate, tricalcium oxide and silicate oxide. In addition there are few other mineral oxides which are responsible for the chemical and physical properties of the aggregate. The powder consists of fine hydrophilic particles which set in the presence of moisture. Hydration of the powder results in a colloidal gel which solidifies to a hard structure in less than $4 \mathrm{hrs}$ and has compressive strength equal to amalgam. ${ }^{4}$

MTA has been advocated as an apexification material because it permits an adequate seal of the canal ${ }^{8}$, prevents bacterial leakage, good marginal adaptation ${ }^{9}$ and biocompatibility. ${ }^{6}$

MTA set in $4 \mathrm{hrs}$ due to this canal was obturated in second appointment. Studies have supported two-step apexification over one step procedure. ${ }^{2}$ In this case $5 \mathrm{~mm}$ of MTA apical plug was formed. Hachmeister 
et al. reported that a 5-mm apical plug is apropriate to prevent infiltration of fluids in the canal. ${ }^{10}$ This clinical case demonstrates that MTA apexification eliminate the lengthy conventional procedure.

\section{CONCLUSION}

Apexification with MTA is a boon for effective management of teeth with open apex. This procedure is predictable and less time consuming.

\section{REFERENCES}

1. Hachmeister DR, Schindler WG, Walder WA and Thomas DD. The sealing ability and retention characteristics of mineral trioxide aggregate in a model of apexification. $\mathrm{J}$ Endod 2002;28(5):386-90.

2. Matt GD, Thorpe JR, Strother JM and McClanahan SB. Comparative study of white and gray mineral trioxide aggregate stimulating a one-or two step apical barrier technique. J Endod 2004;30(12):876-879.

3. Torabinejad M, Chivian N. Clinical application of mineral trioxide aggregate. J Endod 1999;25:197-205.
4. TorabinejadM, WatsonTF, Pitt FordTR. Sealing ability of a mineral trioxide aggregate when used as a root end filling material. J Endod 1993;19(12):591-595.

5. Asgray S, Parirokh M, Eqhbal MJ and Brink F. Chemical differences between white and gray mineral trioxide aggregate. J Endod 2005;31(2):101-103.

6. Koh ET, McDonald F, Ford TRP and Torbinejad M. Cellular response to mineral trioxide aggregate. J Endod 1998;24(8):543-547.

7. Perez AL, Spears R, Gutmann JL and Opperman La. Osteoblasts and MG-63 osteosarcoma sscells behave differently when in contact with ProRoot MTA and white MTA. Int Endod J 2003;36:564-570.

8. Estrela C, Bammann LL, Pimenta FC, PecoraJD. Control of microorganisms in vitro by calcium hydroxide pastes. Int Endod J 2001;34:341.

9. TorabinejadM, Smith PW, Kattering JD, Pitt FordTR. Comparative investigation of marginal adaptation of MineralTrioxide Aggregate and other commonly used rootend filling materials. J Endod 1995;21:295-9.

10. Hachmeister DR, Schindler WG, Walker WA III, Thomas DD. The sealing ability and retention characteristics of mineral trioxide aggregate in a model of apexification. $\mathbf{J}$ Endod 2002;28:386-90. 